\title{
Needing help to use a toilet was associated with faecal incontinence at 3 months in new onset faecal incontinence after stroke
}

\author{
Harari D, Coshall C, Rudd, AG, et al. New-onset fecal incontinence after stroke: prevalence, natural history, risk factors, \\ and impact. Stroke 2003;34:144-50.
}

QUESTION: In patients with new onset faecal incontinence after stroke, which
prognostic factors are associated with bowel continence status at 3 months of follow up?

Design

Inception cohort followed up for 3 years.

\section{Setting}

South London, UK.

\section{Patients}

1468 patients without pre-existing faecal incontinence, who survived a first in a lifetime stroke were recruited from the community based South London Stroke Register. 846 patients $(53 \%$ men) were included in the primary analysis at 3 months (412 patients had died, and data for 210 patients were not available).

\section{Assessment of prognostic factors}

Data were collected in the acute stroke phase (within the first $10 \mathrm{~d}$ ) by research registrars, and at 3 months, 1 year, and then annually by face to face interviews with a trained field worker. When reliable answers could not be obtained directly from patients (because of cognitive impairment, communication difficulties, or severe illness), the information was sought from family, caregivers, nursing home or hospital staff, or community health workers. Medical information was also obtained from hospital and general practitioner records. Associations between faecal incontinence and prognostic factors were assessed in a cross sectional analysis using bivariate and multivariate statistical methods.

\section{Main outcome measure}

Bowel continence status measured by the Barthel Index bowel subscore at 7-10 days, 3 months, and 1, 2, and 3 years.

\section{Main results}

Prevalence of faecal incontinence after stroke was 30\% at $7-10$ days, $11 \%$ at 3 months and 1 year, and $15 \%$ at 3 years. $35 \%$ of patients with faecal incontinence at 3 months were continent by 1 year; $63 \%$ of patients with faecal incontinence at 1 year had been continent at 3 months. Of patients who were continent at 3 months, $6 \%$ became incontinent at 1 year.

Multivariate analysis of acute stroke characteristics showed that initial urinary incontinence (odds ratio [OR] 6.2, 95\% CI 3.2 to 11.9 ) and visual and/or sensory neglect (OR 1.9, CI 1.0 to 3.5) were associated with faecal incontinence. After further adjustment for significant clinical and functional variables, needing help to use a toilet (OR 3.5, CI 1.4 to 17.3) and use of anticholinergic drugs (OR 3.1, CI 1.1 to 10.2) were independent prognostic factors for faecal incontinence.

\section{Conclusion}

New onset faecal incontinence in stroke survivors was common but may be transient. Needing help to use a toilet and use of anticholinergic drugs were associated with new onset faecal incontinence at 3 months after stroke.

\section{COMMENTARY}

The study by Harari et al enhances previous research on faecal incontinence in patients with stroke as it includes only patients with newly acquired faecal incontinence after stroke. Most importantly for healthcare professionals, this study assessed the contribution of potentially modifiable risk factors such as functional difficulties with toilet access and use of drugs that could lead to constipation with overflow.

Bowel continence status was assessed using a question from the Barthel Index. Patients were classified into 1 of 3 categories: "never" losing bowel control, "occasional accident," or "all the time." Although the validity of the Barthel Index as a functional measure has been shown, it may be limited in its ability to assess faecal incontinence. This limitation is apparent when one considers that a patient may have had only 1 faecal incontinence episode and yet be classified within the faecal incontinent sample. This classification may increase the similarity between the 2 groups (never experiencing faecal incontinence $v$ experiencing faecal incontinence), and thus, any true association would be underestimated. A more sensitive measure of bowel continence would have strengthened this study. Furthermore, the lack of precision within the confidence intervals around the odds ratios for anticholinergic drug use and needing help with toilet use reflects substantial variability of the results. Thus, these results should be regarded as preliminary.

The results of this study are relevant to nurses, nurse practitioners, and clinical nurse specialists working with patients on stroke rehabilitation units and complex continuing care or long term care facilities. Because $>10 \%$ of patients with stroke will have faecal incontinence, it is important to identify those individuals at greatest risk. In particular, the results show that faecal incontinence may be transient and modifiable. This will assist nurses to develop care plans to strengthen functional ability, particularly with respect to toileting. The findings also emphasise the importance of assessing modifiable risk factors in addition to clini$\mathrm{cal}$ and demographic factors.

Nancy J Martin, RN, PhD Director, Research and Performance Metrics Grand River Hospital Kitchener, Ontario, Canada
Sources of funding:

Action Research, Northern and Yorkshire Research and Development Cardiovascular Diseases Programme, Charitable Foundation of Guy's and St Thomas' Hospital, and Stanley Thomas Johnson Foundation.

For correspondence: Dr D Harari, Department of Healthcare of the Elderly, Guy's and St Thomas' Hospitals Trust, London, UK. danielle.harari@ kcl.ac.uk 